\title{
Theory of impurity resonant tunnel magnetoresistance
}

\author{
L. Sheng, ${ }^{1}$ D. Y. Xing, ${ }^{1}$ and D. N. Sheng ${ }^{2}$ \\ ${ }^{1}$ National Laboratory of Solid State Microstructures, Nanjing University, Nanjing 210093, China \\ ${ }^{2}$ Department of Physics and Astronomy, California State University, Northridge, California 91330, USA
}

(Received 10 December 2003; published 23 April 2004)

\begin{abstract}
The Green's-function approach is developed to study the tunneling magnetoresistance (TMR) in magnetic tunnel junctions. The total conductance explicitly derived is a sum over electron direct tunneling and tunneling via impurities in the barriers. It is found that, on resonance, even a single impurity may result in inversion of TMR in small-area junctions. The conditions, under which large inverse TMR may occur, are investigated. The statistical distribution and fluctuations of TMR in sign and magnitude varying with impurity density and cross section of the junctions are calculated. Our results reproduce essential features of recent experimental measurements on $\mathrm{Ni} / \mathrm{NiO} / \mathrm{Co}$ nanowire junctions.
\end{abstract}

DOI: 10.1103/PhysRevB.69.132414

PACS number(s): 72.25.-b, 72.10.Fk, 73.40.Rw, 75.47.-m

Tunneling magnetoresistance (TMR) in magnetic tunnel junctions with two ferromagnetic (FM) electrodes separated by an insulating layer ${ }^{1-3}$ has been the main focus of many research activities in recent years because of its promising device application. The TMR represents the effect that the tunneling resistance changes as the magnetizations of the two electrodes change their relative orientation under an applied magnetic field. It originates from the spin polarization of conduction electrons in the FM electrodes, ${ }^{4,5}$ and may also be strongly affected by other electronic properties of the tunnel junctions. ${ }^{6-10}$

In general, when both FM electrodes have spin polarization of the same sign [positive for Fe, Co, and Ni (Ref. 11)], the conductance $\left(G_{\mathrm{P}}\right)$ for parallel $(\mathrm{P})$ alignment is greater than that $\left(G_{\mathrm{AP}}\right)$ for antiparallel (AP) alignment, i.e., the TMR ratio defined as $\mathrm{TMR}=\left(G_{\mathrm{P}}-G_{\mathrm{AP}}\right) /\left(G_{\mathrm{P}}+G_{\mathrm{AP}}\right)$ is positive. Very interestingly, Tsymbal et al. and Sokolov et al. ${ }^{12}$ observed both positive and negative TMR with ratio ranging from -0.14 to 0.17 using a number of $\mathrm{Ni} / \mathrm{NiO} / \mathrm{Co}$ nanowire junctions of cross section smaller than $0.01 \mu \mathrm{m}^{2}$. The inversion of TMR was ascribed to resonant tunneling via the impurity states in $\mathrm{NiO}$. Some of the experimental data were fitted reasonably well by a one-dimensional (1D) Lorentz conductance formula. The authors also introduced Anderson disorder and inelastic scattering to reproduce numerically the measured statistical distribution of TMR by modeling the barrier as a $8 \times 8 \times 8$ tight-binding atomic structure. However, too small cross section of barrier used in the calculation (three orders smaller than the experimental value) makes the result not satisfactory. According to the experiments, ${ }^{12}$ the distribution of the TMR should be sensitive to the sample size.

The above experiments ${ }^{12}$ lead to an important topic in spin-polarized electron transport. Impurity effect on the TMR has previously been studied both experimentally ${ }^{13}$ and theoretically. ${ }^{14-17}$ It has also been predicted that electron resonant tunneling via impurities may cause inversion of the TMR. ${ }^{16,17}$ However, these latter works were mainly based upon 1D approximation ${ }^{16}$ or numerical computation from 3D tight-binding model. ${ }^{17} \mathrm{~A}$ transparent analytical theory for the inversion of TMR that is applicable to realistic 3D junctions is highly desirable.
In this work, the impurity effect on TMR is reexamined based upon a nonperturbative Green's-function approach, in which the electron direct tunneling and tunneling via impurities are treated on an equal footing. A new conductance formula of Lorentz form for resonant tunneling via impurities is explicitly derived. It is found that, on resonance, even a single impurity in the barrier can change the TMR dramatically in a small-area junction (cross section $\lesssim 0.01 \mu \mathrm{m}^{2}$ ), and in particular, may result in a rather large inverse TMR. The statistical distribution and fluctuations of TMR in sign and magnitude varying with impurity density and cross section of the junctions are calculated. The results can explain consistently the distribution of TMR observed in small-area $\mathrm{Ni} / \mathrm{NiO} / \mathrm{Co}$ nanowire junctions, ${ }^{12}$ as well as the very small TMR of 0.01 or less observed earlier in larger-area $\mathrm{Ni} /$ $\mathrm{NiO} / \mathrm{Co}$ junctions. ${ }^{18}$

Let us consider a nanowire junction system consisting of two FM electrodes separated by an insulating barrier. The cross section of the system is a square of side $W$ and the width of the barrier is $L$. The current flows along the $z$ axis and the two FM electrodes are located at $z<0$ and $z>L$, respectively. For simplicity, we assume that the system is symmetric: the two electrodes are made of the same FM material. In the nanowire junction $(0 \leqslant x \leqslant W$ and $0 \leqslant y$ $\leqslant W$ ) without disorder, the single-particle model Hamiltonian can be written as $H_{0}(\sigma, \mathbf{r})=-\nabla^{2} / 2 m_{1}^{*}-\sigma J$ for $z$ $<0,-\nabla^{2} / 2 m_{2}^{*}+U_{0}$ for $0<z<L$, and $-\nabla^{2} / 2 m_{1}^{*}-\eta \sigma J$ for $z>L$. Here $\sigma=+$ and - are the spin index with respect to a fixed reference direction, $\eta=1$ and -1 correspond to $\mathrm{P}$ and AP alignments, respectively. Different effective masses $m_{1}^{*}$ and $m_{2}^{*}$ are used to describe the possibly quite different electron dispersion relations in the ferromagnets and in the barrier. $J$ stands for the exchange-split energy in the ferromagnets ${ }^{6}$ and $U_{0}$ is the barrier height.

The unperturbed wave function for an electron of energy $E$ can be written in a variable-separated form: $\psi_{\sigma}(E ; \mathbf{r})$ $=\chi_{n}(x, y) \varphi_{n \sigma}(z), \quad$ where $\quad \chi_{n}(x, y)=(2 / W) \sin \left(n_{x} \pi x /\right.$ $W) \sin \left(n_{y} \pi y / W\right)$ with $n=\left(n_{x}, n_{y}\right)$ representing quantum numbers in the lateral directions, and $\varphi_{n \sigma}(z)$ is the longitudinal wave function. For a wave incident from the left electrode, 
the outgoing wave function in the right electrode is given by $\varphi_{n \sigma}(z)=t_{n \sigma} \sqrt{v_{n \sigma}^{(\mathrm{L})} / v_{n \sigma}^{(\mathrm{R})}} e^{i k_{n \sigma}^{(\mathrm{R})}(z-L)}$ with the transmission amplitude

$$
t_{n \sigma}=t_{n \sigma}^{(\mathrm{L})} t_{n \sigma}^{(\mathrm{R})} e^{-\alpha_{n} L} I_{n \sigma} .
$$

Here, $t_{n \sigma}^{(A)}=2 \sqrt{i \nu_{n} v_{n \sigma}^{(A)}} /\left(v_{n \sigma}^{(A)}+i \nu_{n}\right), r_{n \sigma}^{(A)}=\left(v_{n \sigma}^{(A)}-i \nu_{n}\right) /\left(v_{n \sigma}^{(A)}\right.$ $\left.+i \nu_{n}\right)$, and $I_{n \sigma}=\left(1-r_{n \sigma}^{(\mathrm{L})} r_{n \sigma}^{(\mathrm{R})} e^{-2 \alpha_{n} L}\right)^{-1}$ with $A=\mathrm{R}(\mathrm{L})$ standing for the left (right) electrode. $\alpha_{n}=\left[2 m_{2}^{*}\left(U_{0}-E\right)\right.$ $\left.+k_{\|_{n}}^{2}\right]^{1 / 2}$ is the decay exponent in the barrier, and $\nu_{n}$ $=\alpha_{n} / m_{2}^{*} \quad$ with $\quad k_{\| n}=(\pi / W)\left(n_{x}^{2}+n_{y}^{2}\right)^{1 / 2} . \quad k_{n \sigma}^{(\mathrm{L})}=\left[2 m_{1}^{*}(E\right.$ $\left.+\sigma J)-k_{\|_{n}}^{2}\right]^{1 / 2}, \quad k_{n \sigma}^{(\mathrm{R})}=\left[2 m_{1}^{*}(E+\eta \sigma J)-k_{\|_{n}}^{2}\right]^{1 / 2}, \quad$ and $v_{n \sigma}^{(A)}$ $=k_{n \sigma}^{(A)} / m_{1}^{*}$ are the longitudinal wave vectors and velocities of electrons in the electrodes.

Next we consider $N_{\text {I }}$ attractive impurities in the barrier. Their potential is assumed to be $V(\mathbf{r})=-\sum_{i} V_{i} e^{-\left|\mathbf{r}-\mathbf{R}_{i}\right| / \ell}$, where $\mathbf{R}_{i}=\left(X_{i}, Y_{i}, Z_{i}\right)$ is the position of impurity $i$. For simplicity, we will take the point-impurity limit $\ell \rightarrow 0$. Here, to focus on the inversion of TMR, we will not consider the interaction or magnetic impurities, which might also play an important role in realistic junctions. ${ }^{13,14}$ According to general scattering theory, ${ }^{19}$ the scattered electron wave function $\Psi_{\sigma}(E ; \mathbf{r})$ is determined by

$$
\begin{aligned}
\Psi_{\sigma}(E ; \mathbf{r})= & \psi_{\sigma}(E ; \mathbf{r})-\sum_{i} V_{i} \int d^{3} r^{\prime} G_{\sigma}\left(E ; \mathbf{r}, \mathbf{r}^{\prime}\right) \\
& \times e^{-\left|\mathbf{r}^{\prime}-\mathbf{R}_{i}\right| / \ell} \Psi_{\sigma}\left(E ; \mathbf{r}^{\prime}\right) .
\end{aligned}
$$

Here, $G_{\sigma}\left(E ; \mathbf{r}, \mathbf{r}^{\prime}\right)$ is the retarded Green's function, which can be calculated in a similar way to calculating the Green's function for a thin film. ${ }^{20}$ In the second term $\Psi_{\sigma}\left(E ; \mathbf{r}^{\prime}\right)$ is not singular and can be replaced by $\Psi_{\sigma}\left(E ; \mathbf{R}_{i}\right)$ in the $\ell \rightarrow 0$ limit. Setting $\mathbf{r}$ on both sides of Eq. (2) to $\mathbf{R}_{i}$, we obtain $N_{\text {I }}$ selfconsistent equations for determining $\Psi_{\sigma}\left(E ; \mathbf{R}_{i}\right)$. By substituting the solutions back into Eq. (2), the wave function $\Psi_{\sigma}(E ; \mathbf{r})$ can be derived. In order to obtain an explicit result, we confine ourselves to the case, where the average distance between impurities is much greater than $L$. In this case, the inter-impurity tunneling is very small on an average and may be neglected. In general, the impurity scattering leads to a mixing of different channels. The transmission amplitude $\tau_{n \sigma, m \sigma}$ from channel $n \sigma$ to channel $m \sigma$ is derived to be

$$
\begin{aligned}
\tau_{n \sigma, m \sigma}= & t_{n \sigma} \delta_{n m}+\sum_{i}\left(\frac{8 \pi V_{i} \ell^{3} I_{n \sigma} I_{m \sigma}}{\sqrt{\nu_{n} \nu_{m}}\left[1+V_{i} P_{i \sigma}\left(E ; \mathbf{R}_{i}\right)\right]}\right) \\
& \times \chi_{n}\left(X_{i}, Y_{i}\right) \chi_{m}\left(X_{i}, Y_{i}\right) t_{n \sigma}^{(L)} t_{m \sigma}^{(R)} \\
& \times\left(e^{-\alpha_{n} d_{i}^{(L)}}-r_{n \sigma}^{(R)} e^{-\alpha_{n}\left(2 L-d_{i}^{(L)}\right)}\right) \\
& \times\left(e^{-\alpha_{m} d_{i}^{(R)}}-r_{m \sigma}^{(L)} e^{-\alpha_{m}\left(2 L-d_{i}^{(R)}\right)}\right),
\end{aligned}
$$

where $P_{i \sigma}(E ; \mathbf{r})=\int d^{3} r^{\prime} G_{\sigma}\left(E ; \mathbf{r}, \mathbf{r}^{\prime}\right) e^{-\left|\mathbf{r}^{\prime}-\mathbf{R}_{i}\right| / \ell}$, and $d_{i}^{(\mathrm{L})}$ $=Z_{i}\left(d_{i}^{(\mathrm{R})}=L-Z_{i}\right)$ is the distance between impurity $i$ and the left (right) interface. The bound state on impurity $i$ corresponds to a pole of the Green's function, of which the energy is determined by $1+V_{i} \operatorname{Re}\left[P_{i \sigma}\left(E ; \mathbf{R}_{i}\right)\right]=0$. We find that there exists a bound state on the impurity at energy $E_{i}^{\mathrm{I}}$
$=U_{0}-\Delta_{i}$ if $\lambda_{i} \equiv \sqrt{2 m_{2}^{*} V_{i}} \ell>1$, where $\Delta_{i}=\left(1-\lambda_{i}^{-1}\right)^{2} V_{i}$ is the bound energy. For the denominator of the second term in Eq. (3), expanding its real part to the leading order in $(E$ $\left.-E_{i}^{\mathrm{I}}\right)$, we obtain

$$
\tau_{n \sigma, m \sigma}=t_{n \sigma} \delta_{n m}+\sum_{i} \frac{2 \gamma_{i n \sigma}^{(\mathrm{L})}\left(E_{i}^{\mathrm{I}}\right) \gamma_{i m \sigma}^{(\mathrm{R})}\left(E_{i}^{\mathrm{I}}\right)}{E-E_{i}^{\mathrm{I}}+i\left(\Gamma_{i \sigma}^{(\mathrm{L})}+\Gamma_{i \sigma}^{(\mathrm{R})}\right)},
$$

where

$$
\begin{aligned}
\gamma_{i n \sigma}^{(A)}(E)= & \sqrt{\frac{\pi}{\alpha_{n}}}\left(\frac{2 \Delta_{i}}{m_{2}^{*}}\right)^{1 / 4} I_{n \sigma} \chi_{n}\left(X_{i}, Y_{i}\right) \\
& \times t_{n \sigma}^{(A)}\left(e^{-\alpha_{n} d_{i}^{(A)}}-r_{n \sigma}^{(\bar{A})} e^{-\alpha_{n}\left(2 L-d_{i}^{(A)}\right)}\right),
\end{aligned}
$$

and $\Gamma_{i \sigma}^{(A)}=\Sigma_{n}\left|\gamma_{i n \sigma}^{(A)}\left(E_{i}^{\mathrm{I}}\right)\right|^{2}$. Here $\bar{A}$ represents the electrode other than $A$. The point-impurity limit of $\ell \rightarrow 0$ and $V_{i} \rightarrow \infty$ has been taken under the constraint of fixed $\Delta_{i}$. The tunneling conductance can be calculated using the LandauerBüttiker formula ${ }^{21,22} \quad G=(e / 2 \pi \phi) \Sigma_{\sigma} \int d E T_{\sigma}(E)\left[f_{0}(E\right.$ $\left.-e \phi)-f_{0}(E)\right]$, where $\phi$ is the bias voltage and $T_{\sigma}(E)$ $=\Sigma_{n m}\left|\tau_{n \sigma, m \sigma}\right|^{2}$ is the total transmission coefficient.

In Eq. (4), the first term represents the direct tunneling in the absence of impurities, and the second term stands for the resonant tunneling via the impurities. Let us first consider the simplest case of single impurity, and omit the impurity index $i$ temporarily. For $E \simeq E^{\mathrm{I}}$, the crossing terms in $\left|\tau_{n \sigma, m \sigma}\right|^{2}$ between the direct and resonant transmission amplitudes are proportional to $E-E^{\mathrm{I}}$, and so make no contribution to $T_{\sigma}(E)$. It then follows that $T_{\sigma}(E)=T_{\sigma}^{\mathrm{D}}(E)+T_{\sigma}^{\mathrm{R}}(E)$, where $T_{\sigma}^{\mathrm{D}}(E)=\Sigma_{n}\left|t_{n \sigma}\right|^{2}$ and $T_{\sigma}^{\mathrm{R}}(E)=4 \Gamma_{\sigma}^{(\mathrm{L})} \Gamma_{\sigma}^{(\mathrm{R})} /\left[\left(E-E^{\mathrm{I}}\right)^{2}+\left(\Gamma_{\sigma}^{(\mathrm{L})}\right.\right.$ $\left.\left.+\Gamma_{\sigma}^{(\mathrm{R})}\right)^{2}\right]$. It is interesting to see that the present resonant transmission coefficient $T_{\sigma}^{\mathrm{R}}(E)$ has the same form as the 1D formula, Eq. (2) in Ref. 12, where $\Gamma_{\sigma}^{(A)}(E)$ was a phenomenological parameter and assumed proportional to $\rho_{\sigma}^{(A)} e^{-2 \kappa d^{(A)}}$ with $\kappa$ the decay constant and $\rho_{\sigma}^{(A)}$ the density of states in electrode $A$. The present Eq. (4) is much more general than Eq. (2) of Ref. 12. It is suitable for realistic 3D tunnel junctions, where for a given energy many channels contribute to the transport, and includes a competition between the direct and resonant tunneling processes. The conductance formula in Ref. 12 has been used to fit with some of experimental data for the bias voltage dependence of TMR with prefactors in $\Gamma_{\sigma}^{(\mathrm{L})}$ and $\Gamma_{\sigma}^{(\mathrm{R})}$ as adjustable parameters. Here, we will not repeat this fitting. Instead, we discuss a few of new results implied by Eq. (4).

The calculated TMR at zero-bias voltage as a function of the impurity position $\mathbf{R}$ is plotted in Fig. 1(a) for $E_{\mathrm{F}}=E^{\mathrm{I}}$. The region of $Z>0.5 L$ (or $X>0.5 W$ ) is symmetric to that in Fig. 1(a). To be close to the experimental condition, ${ }^{12}$ the size of the junction is taken to be $L=1.5 \mathrm{~nm}$ and $W$ $=80 \mathrm{~nm}$. The electron effective mass in the FM electrodes is assumed equal to the bare electron mass $m_{1}^{*}=m_{e}$. For insulating $\mathrm{NiO}$, the experimental width of the unoccupied conduction band is $18 \mathrm{eV}^{23}$ We estimate the effective mass $m_{2}^{*} \simeq 0.2 m_{e}$ by expanding a tight-binding dispersion relation at the band bottom. In Fig. 1(a), we see that the TMR has no 



FIG. 1. (a) TMR as a function of normalized impurity position $X / W$ and $Z / L$ for $Y=0.5 W$ and $E_{\mathrm{F}}=E^{\mathrm{I}}$, and (b) TMR as a function of $E_{\mathrm{F}}-E^{\mathrm{I}}$ and $Z / L$ for $X=Y=0.5 W$. Here $J=1.6 \mathrm{eV}, E_{\mathrm{I}}$ $=2 \mathrm{eV}$, and $U_{0}=4 \mathrm{eV}$ for both figures. visible dependence on the lateral impurity coordinate $X$ (and $Y$ by symmetry), except on the boundary where $\chi_{n}(X, Y)$ vanishes. If the impurity is close to the middle plane of the barrier $(Z \simeq 0.5 L)$, for $\mathrm{P}$ alignment we have $\Gamma_{\sigma}^{(\mathrm{L})} \simeq \Gamma_{\sigma}^{(\mathrm{R})}$ and hence $T_{\sigma}^{\mathrm{R}}(E) \simeq 1$. Since its maximum value is $1, T_{\sigma}^{\mathrm{R}}(E)$ for $\mathrm{P}$ alignment must be greater than that for AP alignment, yielding a positive TMR. If $Z$ deviates from $Z=0.5 L$ by a sufficiently long distance, assumed in the left half of the barrier, we have $\Gamma_{\sigma}^{(\mathrm{L})} \gg \Gamma_{\sigma}^{(\mathrm{R})}$ so that $T_{\sigma}^{\mathrm{R}}(E) \simeq 4 \Gamma_{\sigma}^{(\mathrm{R})} / \Gamma_{\sigma}^{(\mathrm{L})}$. The total resonant transmission coefficient $\Sigma_{\sigma} T_{\sigma}^{\mathrm{R}}(E)$ will be denoted as $T_{\mathrm{P}}^{\mathrm{R}}(E)$ for $\mathrm{P}$ alignment and $T_{\mathrm{AP}}^{\mathrm{R}}(E)$ for $\mathrm{AP}$ alignment. Their difference can be obtained as $T_{\mathrm{P}}^{\mathrm{R}}(E)-T_{\mathrm{AP}}^{\mathrm{R}}(E)$ $\simeq-4\left(\Gamma_{\uparrow}^{(\mathrm{R})}-\Gamma_{\downarrow}^{(\mathrm{R})}\right)\left(\Gamma_{\uparrow}^{(\mathrm{L})}-\Gamma_{\downarrow}^{(\mathrm{L})}\right) / \Gamma_{\uparrow}^{(\mathrm{L})} \Gamma_{\downarrow}^{(\mathrm{L})}$, where subscript $\uparrow$ $(\downarrow)$ stands for the majority (minority) spin in the corresponding FM electrode. In general, $\Gamma_{\uparrow}^{(A)}>\Gamma_{\downarrow}^{(A)}$ because more channels contribute to the summation over $n$ of $\Gamma_{\uparrow}^{(A)}$ in the majority-spin band. It follows that $T_{\mathrm{AP}}^{\mathrm{R}}(E)>T_{\mathrm{P}}^{\mathrm{R}}(E)$. Thus, the spin-dependent resonant tunneling via the impurity gives rise to a negative $\mathrm{TMR}$ in the region of $0.27<Z / L<0.45$. With further decreasing $Z, T_{\sigma}^{\mathrm{R}}(E)$ decreases exponentially, so that $T_{\sigma}^{\mathrm{D}}(E)$ dominates and the TMR returns to positive for $Z / L<0.27$. The calculated TMR as a function of $Z / L$ and $E_{\mathrm{F}}-E^{\mathrm{I}}$ is shown in Fig. 1(b). It is clear that the Fermi energy must be close enough to the impurity energy, e.g., $\left|E_{\mathrm{F}}-E^{\mathrm{I}}\right|$ $\$ 8 \mathrm{meV}$ for the parameters chosen, to obtain an inverse TMR.

In Fig. 2, the zero-bias TMR on resonance is plotted as a function of $Z / L$ for different value of barrier height $U_{0}$. An important finding is that there exists a critical value of barrier

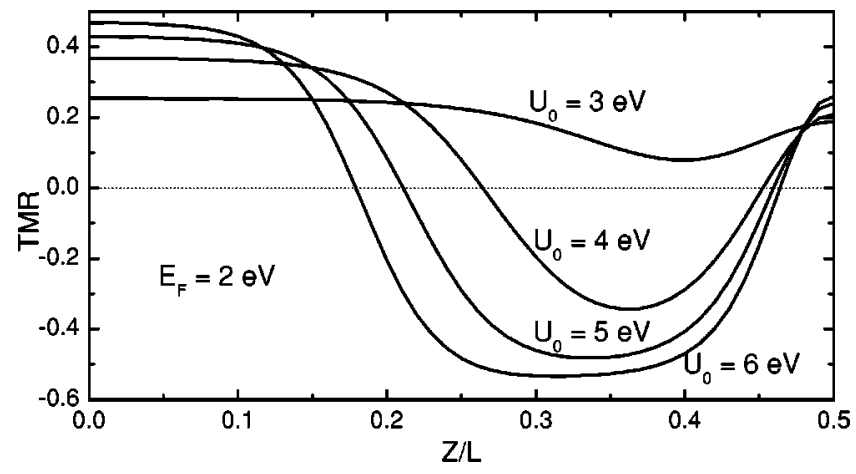

FIG. 2. TMR as a function of normalized impurity position $Z / L$ for different barrier heights. Here, $X=Y=0.5 \mathrm{~W}$ and the other parameters are the same as in Fig. 1(a). height below which TMR is always positive. This behavior can be understood by the following argument. As mentioned above, negative TMR may possibly occur only when the impurity is away from $Z=0.5 L$. The resonant transmission can be roughly estimated as $T_{\sigma}^{\mathrm{R}}(E) \propto e^{-2 \alpha_{0}(L-2 Z)}$ with a prefactor of order 1 , where $\alpha_{0}=\left[2 m_{2}^{*}\left(U_{0}-E\right)\right]^{1 / 2}$. At the same time, the direct transmission is roughly $T_{\sigma}^{\mathrm{D}}(E) \propto e^{-2 \alpha_{0} L}$ and contains a big prefactor of the same order of magnitude as the number of lateral states below $E_{\mathrm{F}}$ due to the summation over $n$ in $T_{\sigma}^{\mathrm{D}}(E)$. If the barrier height $U_{0}$ is higher than but very close to $E_{\mathrm{F}}, \alpha_{0}$ is small and hence the exponential factors in the two transmission coefficients are close to each other. In this case, the direct tunneling dominates and the TMR is positive for all the values of $Z / L$. With increasing $U_{0}, \alpha_{0}$ increases and the exponential factor in $T_{\sigma}^{\mathrm{R}}(E)$ becomes much greater than that in $T_{\sigma}^{\mathrm{D}}(E)$. As soon as the disproportion of the two exponential factors prevails over the big prefactor of $T_{\sigma}^{\mathrm{D}}(E)$, the resonant tunneling dominates and an inverse TMR occurs. The positive TMR at either small or large $Z / L$ and the existence of critical $U_{0}$ are two typical examples that can not be understood by using the simple formula of Ref. 12. Systems with one or a few impurities actually represent an optimal case, where largest inverse TMR may probably be observed.

After clarifying the physical picture of sign inversion of TMR in the single-impurity case, we now turn to studying the many-impurity case, which very likely corresponds to the real magnetic tunnel junctions used in the experiments. ${ }^{12,18}$ The total transmission coefficient is given by $T_{\sigma}(E)$ $=T_{\sigma}^{\mathrm{D}}(E)+\Sigma_{i} T_{i \sigma}^{\mathrm{R}}(E)$ with $T_{i \sigma}^{\mathrm{R}}(E)$ as the transmission coefficient via impurity $i$. For an ensemble of identical junctions, the positions and energies of the impurities are random, and so $T_{i \sigma}^{\mathrm{R}}(E)$ is a random variable. For large $N_{\mathrm{I}}$, the asymptotic behavior of the total transmission coefficient $T_{\sigma}(E)$, as a sum of individual $T_{i \sigma}^{\mathrm{R}}(E)$, can be analyzed by applying the central limit theorem. ${ }^{24}$ As a result, the variance of the TMR is found to decrease as $1 / N_{\mathrm{I}}$; i.e.,

$$
\left[\left\langle\mathrm{TMR}^{2}\right\rangle-\langle\mathrm{TMR}\rangle^{2}\right] \propto \frac{1}{n_{\mathrm{I}} L W^{2}},
$$

with $n_{\mathrm{I}}$ as the impurity density in the barrier. It indicates that the TMR has small fluctuations around its average in large area or dirty tunnel junctions, while larger negative or positive TMR may be observed in small-area or high-purity junc- 


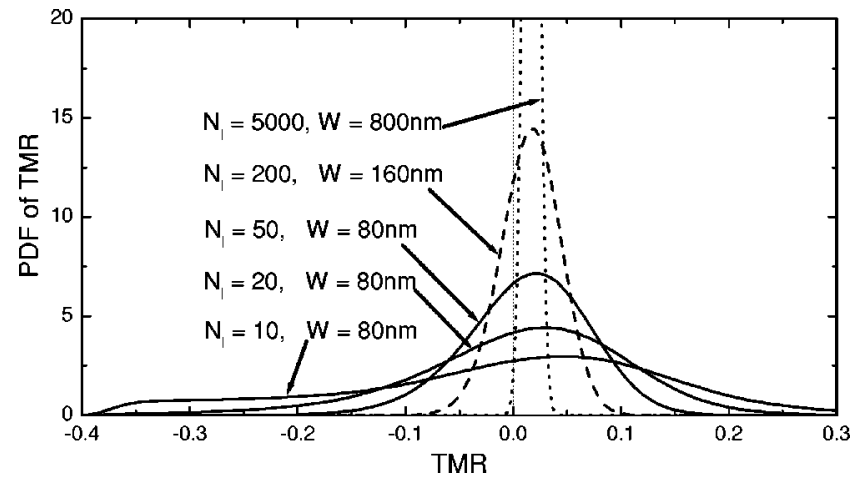

FIG. 3. PDF of TMR for some values of impurity number and lateral size of the tunnel junction. Here $w=10 \mathrm{meV}$ and other parameters are the same as in Fig. 1(a).

tions. This conclusion is very general, independent of the specific form of the distribution function of impurities. For given distribution function of impurity positions and energies, we can calculate the probability distribution function (PDF) of TMR quantitatively. Since information about the impurity distribution is not available currently, we simply assume that the positions of impurities are random throughout the barrier, and their energies are distributed randomly in a narrow impurity band of width $w$ around $E_{\mathrm{F}}$. The calculated PDF of TMR are shown in Fig. 3. The three solid lines describe how the PDF changes with $N_{\text {I }}$ for fixed cross section. The PDF for $N_{\mathrm{I}}=50$ is similar to the experimental TMR distribution, Fig. 1 of Ref. 12. For this $N_{\mathrm{I}}$, the density of impurity bound states is $n_{\mathrm{I}} \simeq 0.5 \times 10^{25} / \mathrm{m}^{3}$, being of the correct order compared with the carrier density $10^{25} / \mathrm{m}^{3}$ in the barrier deduced from the Mott-Schottky analysis. ${ }^{12}$ Therefore, in the present theory, the experimental TMR distribution is attributed to the randomly distributed attractive impurities, which is a clear and straightforward picture. In Fig. 3, the three curves with $N_{\mathrm{I}}=50,200$, and 5000 illustrate the variation of the PDF with changing the cross section $W^{2}$ of the magnetic junctions for fixed impurity density $n_{\mathrm{I}}$ $\left(=N_{\mathrm{I}} / L W^{2}\right)$. With increasing the cross section, the PDF narrows around an average $\mathrm{TMR} \simeq 0.02$. This explains theoretically why very small TMR were observed in large-area $\mathrm{Ni} /$ $\mathrm{NiO} / \mathrm{Co}$ tunnel junctions. ${ }^{18}$

D.Y.X. thanks the support from the State Key Program for Basic Research of China, and the support from the National Natural Science Foundation of China under Grant No. 10374046. D.N.S. was supported by ACS-PRF Grant No. 36965-AC5, Research Corporation Grant No. CC5643, the NSF Grant Nos. DMR-00116566 and DMR-0307170, and the KITP at Santa Barbara through Grant No. PHY99-07949.
${ }^{1}$ J.S. Moodera, L.R. Kinder, T.M. Wong, and R. Meservey, Phys. Rev. Lett. 74, 3273 (1995).

${ }^{2}$ T. Miyazaki and N. Tezuka, J. Magn. Magn. Mater. 139, 231 (1995).

${ }^{3}$ B. Doudin, G. Redmond, S.E. Gilbert, and J.-Ph. Ansermet, Phys. Rev. Lett. 79, 933 (1997).

${ }^{4}$ M. Julliere, Phys. Lett. 54A, 225 (1975).

${ }^{5}$ S. Maekawa and U. Gäfvert, IEEE Trans. Magn. 18, 707 (1982).

${ }^{6}$ J.C. Slonczewski, Phys. Rev. B 39, 6995 (1989).

${ }^{7}$ S. Zhang, P.M. Levy, A.C. Marley, and S.S.P. Parkin, Phys. Rev. Lett. 79, 3744 (1997); S. Zhang and P.M. Levy, Eur. Phys. J. B 10, 599 (1999).

${ }^{8}$ E.Y. Tsymbal and D.G. Pettifor, J. Phys.: Condens. Matter 9, L411 (1997); Phys. Rev. B 58, 432 (1998).

${ }^{9} \mathrm{Ph}$. Mavropoulos, N. Papanikolaou, and P.H. Dederichs, Phys. Rev. Lett. 85, 1088 (2000).

${ }^{10}$ W.H. Butler, X.-G. Zhang, T.C. Schulthess, and J.M. MacLaren, Phys. Rev. B 63, 054416 (2001).

${ }^{11}$ P.M. Tedrow and R. Meservey, Phys. Rev. B 7, 318 (1973).

${ }^{12}$ E.Y. Tsymbal, A. Sokolov, I.F. Sabirianov, and B. Doudin, Phys. Rev. Lett. 90, 186602 (2003); A. Sokolov, I.F. Sabirianov, E.Y. Tsymbal, B. Doudin, X.Z. Li, and J. Redepenning, J. Appl. Phys. 93, 7029 (2003).

${ }^{13}$ R. Jansen and J.S. Moodera, J. Appl. Phys. 83, 6682 (1998); Appl.
Phys. Lett. 75, 400 (1999); Phys. Rev. B 61, 9047 (2000); S. Tanoue and A. Yamasaki, J. Appl. Phys. 88, 4764 (2000).

${ }^{14}$ F. Guinea, Phys. Rev. B 58, 9212 (1998); A. Vedyayev, D. Bagrets, A. Bagrets, and B. Dieny, ibid. 63, 064429 (2001); H. Sun, K.W. Yu, and Z.Y. Li, ibid. 68, 054413 (2003).

${ }^{15}$ A.M. Bratkovsky, Phys. Rev. B 56, 2344 (1997); Appl. Phys. Lett. 72, 2334 (1998).

${ }^{16}$ R. Jansen and J.C. Lodder, Phys. Rev. B 61, 5860 (2000); J. Inoue, N. Nishimura, and H. Itoh, ibid. 65, 104433 (2002).

${ }^{17}$ E.Y. Tsymbal and D.G. Pettifor, Phys. Rev. B 64, 212401 (2001).

${ }^{18}$ T. Miyazaki and N. Tezuka, J. Magn. Magn. Mater. 151, 403 (1995).

${ }^{19}$ Nouredine Zettili, Quantum Mechanics: Concepts and Applications (Wiley, New York, 2001).

${ }^{20}$ L. Sheng, D.Y. Xing, and Z.D. Wang, Phys. Rev. B 51, 7325 (1995).

${ }^{21}$ R. Landauer, in Nonlinearity in Condensed Matter, edited by A. R. Bishop, D. K. Campbell, P. Kumar, and S. E. Trullinger (Springer, Berlin, 1987), p. 2; T. Christen and M. Büttiker, Europhys. Lett. 35, 523 (1996).

${ }^{22}$ L. Sheng, Y. Chen, H.Y. Teng, and C.S. Ting, Phys. Rev. B 59, 480 (1999).

${ }^{23}$ G.A. Sawatzky and J.W. Allen, Phys. Rev. Lett. 53, 2339 (1984).

${ }^{24}$ W. Feller, An Introduction to Probability Theory and Its Applications (Wiley, New York, 1968), Vol. 1, p. 229. 